\title{
Practice characteristics that lead to 21st century learning outcomes
}

\author{
N. Law, Y. Lee \& A. Chow \\ University of Hong Kong, Hong Kong.
}

\begin{abstract}
The key research question for this study was to ask whether or not innovative teaching practices would lead to the development of learning outcomes essential for preparing the younger generation for the challenges of life in the knowledge society of the 21st century, and if so, how are the pedagogical features related to the different learning outcomes. Preliminary analyses of the case study data collected from the SITES M2 Study in Hong Kong reveal that where the development of more significant learning gains were observed, the cases possess characteristics additional to the criteria defined in the Study for selection of innovation. More importantly, it was found that the impact of the pedagogical practices was not determined simply by the aggregation of characteristics of the practices per se, nor on the technologies used, but on whether 'empowerment' permeates the curriculum goal and process. Further, this paper claims that these affective and socio-cognitive learning outcomes are more important as preparation for lifelong learning in the 21st century than 'knowledge management competencies'.
\end{abstract}

Keywords: Case study; Empowerment; ICT-use; School; learning outcomes, knowledge management competencies, knowledge building, .

\section{Introduction}

A main justification for the widespread introduction of ICT to support teaching and learning across the curriculum has been the development of a new set of competencies to prepare the younger generation for life in the 21st century. The purpose of introducing ICT into the school curriculum can be various and the way this problem has captured the attention of policy makers and the education community as well as the general public at large has witnessed great changes in the last several decades. However, it is undeniable that there is a growing tendency for education policies on ICT in the curriculum to be linked to or made in the context of a wider educational renewal/reform at a national level, e.g. PCAST, 1997; SME, 1997; Ireland, 1999; KME, 2000. This orientation of conceptualising studies of ICT in schools in the context of education reforms has also dominated the research literature, e.g. Kozma \& Schank, 1998. It is also in this context that the Second International Information Technology in Education Study introduced the concept of emerging pedagogical paradigm for the study (Pelgrum \& Anderson, 1999).

Accepted 15 July 2002

Correspondence: Nancy Law, Centre for Information Technology in School and Teacher Education, The University of Hong Kong, Pokfulam Road, Hong Kong SAR, China Email: nlaw@hkusua.hku.hk 


\section{N. Law et al.}

Furthermore, there has also been great consistency in the education reform goals of different countries: preparing students for lifelong learning. Lifelong learning ability is seen both as a demand made on the average citizen of the 21st century as well as what ICT could contribute most in preparing students for.

This paper reports on an exploration of whether technology-using innovative teaching practices would lead to the development of learning outcomes essential for preparing the younger generation for the challenges of life in the knowledge society of the 21st century, using data collected in the SITES M2 study in Hong Kong. As the next phase of SITES, M3 will focus on the international comparison of student achievement in the area of knowledge management competencies (a term that has been used in SITES to refer to the desired 21st century abilities described earlier), this paper will also contribute to examining the connection between the study foci for the two phases of SITES, M2 and M3.

\section{1st century learning outcomes and pedagogical practice characteristics}

Specifications and discussions about the qualities required of individuals to function effectively in the 21st century can be found in many ICT in education masterplans as well as in plans and discussions related to bridging the digital divide (e.g. OECD, 2000). Learning outcomes generally identified to be essential for preparing the younger generation for the challenges of life in the knowledge society include the ability and readiness to engage in lifelong learning, to access and evaluate information, to communicate effectively and to collaborate with others in solving complex open-ended problems, with the appropriate use of technology. Specifically, in the draft plan submitted to IEA for SITES M3, Anderson \& Plomp (2000) identified seven ICT-related knowledge management competencies, which was the term used to refer to the skills and abilities to manage knowledge and to deal with information using ICT:

- retrieve and organise knowledge

- $\quad$ solve complex problems

- collaborate; exchange knowledge; work with experts

- communicate; give persuasive presentation

- construct knowledge products;

- integrate and critically evaluate knowledge

- identify and evaluate secondary effects

The same document, offered a model for linking up the knowledge management competencies as learning outcomes with the pedagogical practice characteristics that are present in schools. Thus, there is a tacit, and perhaps rather persuasive assumption that certain pedagogical features in teaching and learning will lead to the development of particular competencies. A preliminary analysis of the SITES M2 case data collected in Hong Kong indicates that neither the above list of learning outcomes and nor the SITES M2 characterisation for innovative practices (see Kozma \& Anderson in this issue) were able to capture the most important learning outcomes or the pedagogical characteristics of the practices that are most valuable for bringing about the development of 21st century learning outcomes. Further, there is an indication that the relationship between pedagogical practice characteristics and learning outcome is a holistic rather than an atomistic one.

In the remainder of this paper, an attempt will be made to first describe the perceived complexities that distinguish the pedagogical practices in important ways 
beyond the specified innovation characteristics. This will be followed by a description of the observed learning outcomes that go beyond the knowledge management competencies listed above. Finally, this paper will conclude with some suggestions for the design of studies that attempt to link up '21st century' pedagogies with '21st century' learning outcomes.

\section{Learning outcomes gained}

The SITES M2 case study data were analysed to look for evidence of learning outcomes gained through the innovative practices, which are important to citizens of the 21st century. The analysis reveal that while the knowledge management competencies detailed in the SITES M3 design document were observable through the task performance of the students, there is evidence for the learning outcome to be gained in a more holistic manner and that the affective and socio-cognitive dimensions of the learning outcome may possibly be more important than the individual competencies. Here, illustrations of students' learning outcome are taken from a case study conducted in a secondary school. Two pedagogical practices involving different students in this school were studied: one involving the use of a bulletin board to support project-based learning on some Physics topics, and the other involving the use of a multi-age discussion forum to support the learning of the three science subjects, which included the participation of current students as well as past graduates.

\section{Mastering information literacy skills}

As is expected, many students reported gains in information literacy skills through the learning process. This includes using Internet search for locating relevant information, editing reports with WORD and preparing presentations using PowerPoint. Some students even learnt how to apply for a URL and to design a webpage for presenting their projects. The following are some abstracts from the student interviews:

At the beginning all the members in our group did not know how to put things on the Internet and the WebPages design. We learnt these skills in this project.

We went to the public library and found some books on webpage design we do learnt a lot from this.

We also learnt the presentation skills which I think is crucial for my adult life.

\section{Developing critical thinking abilities}

Besides the mastery of new skills, students also reported having sharpened their ability to critically evaluate information and arguments through being confronted by different viewpoints expressed on the forums.

..... In the past when I was confronted with different points of view, I will be in a chaotic situation. But now I will think more in-depth before I post the questions and be more rational in considering other points of view.

Student $L$

...yes, we need to think before posing the message. As this forum is not a synchronised discussion, it is better for us to sharpen our arguments beforehand. For example, you need to think what kinds of counter-arguments people will point out and how you would respond to it. ...... participating in the discussion forum does help me in developing my logical and critical thinking. 


\section{N. Law et al.}

Empowered to learn about the new and unknown

However, in addition to learning gains in knowledge and skills, many students also reported gains along the socio-emotional and socio-cognitive dimensions:

At the beginning I did not think the topic which we are going to investigate was related to Physics. But now I know more about the Butterfly effect.

Compared with other projects that we had done before, we would have some background on the topic [in our earlier projects]. However, the topic in this project was a brand new one; we have not learnt this topic before so we learnt much more in doing this project.

Learning how to learn is not just a matter of cognitive ability, but also the selfconfidence to face the challenge of knowing about something new, and the belief in learning as an incremental process (Seltzer \& Bentley 2001). Thus this kind of learning reported by the students are arguably more important and having more lasting impact.

\section{Empowered to learn from various others}

Another important socio-emotional intelligence involved in effective functioning in a knowledge society is to appreciate and seek knowledge from various others, including subordinates, and not feel threatened. Here, in this practice, both the teachers and students exhibited this kind of learning through the reflections they made on the process:

There was a student asking something in the medical field which I'm not familiar with. However, one of our alumni who is now a doctor gave an answer to this student. Teacher interview

It has definitely had a positive impact on us. It is not easy to find a teacher to help you to solve problems immediately and you can only consult one person at a time. But through the forum, very often you can receive advice from different experts and teachers who can respond to your questions when they are free. It only takes about half an hour or a couple of hours for you to get the response. Student $L$

This openness on the part of both teachers and students, especially the former, to learn from diverse others and to not feel being threatened is seldom observed in traditional classrooms. Yet this is an important precondition to operating effectively as a member of a learning community.

\section{Empowered to contribute as a member of a learning community}

Members of a learning community need to be not only confident about learning from others, but should also be confident and ready to contribute their views and ideas to the community.

Sometimes when I know there is something wrong in some of the posted messages, I will pointed it out and let them know which is the right direction. . . . It is because we are studying in the same school. We are in the same big family. Student $M$

Sometime you get lots of responses. It [the discussion forum] creates a brotherhood culture that encourages us to help each other. Student $M$

In fact, as a contributing member of a learning community, students are not just presenting their own view, but also become intellectually engaged and cognitively challenged in relation to the issues being discussed, motivating further learning: When you see people discussing things and posing ideas on the forum, sometimes it seems that there is something wrong with their arguments. But actually I do not know what's wrong with it and I do want to find out. Therefore, it motivates me to read books on that topic.

Student $N$ 
Empowered to appreciate different viewpoints

Various parties in the school, from students to the school supervisor (the title used in Hong Kong for the chairperson of the school board), also reported witnessing a change in students' attitudes towards different opinions.

It is interesting to see different opinions in the forum. You can see just the opposite opinions on the web. You can compare and think about it. It is fun. Student $M$

There are different points of view in the forum. It provides an environment for nourishing the democratic spirit and the attitude to respect one another even though they have the opposite opinions.

School Supervisor

The learning exhibited above involves a level of understanding of knowledge as socially constructed, as an artefact dissociated from the person generating those views. This level of understanding of level is a sophisticated one, and one which needs to be fostered for effective participation in a knowledge society (Bereiter \& Scardamalia, 1998). Such sophistication in approach to knowledge and understanding is very rarely found in traditional classrooms where generally students would focus on correct understandings.

Empowered through participating in the creation of a learning community

Responses from the students also revealed the kind of awareness and appreciation developed through experiencing the growth and establishment of a learning community as they participate in the discussion forum. There was a strong sense of community exuberating from their descriptions of what the forum meant for them. If there is no discussion forum, it is more difficult for students at different levels to communicate. With the use of [the] forum, messages and news will be delivered in a more effective way. It is just like reading the newspaper and knowing what is happening in the world. You just browse the forum and you know what is happening within the school. Student $L$

This sense of community is exhibited not only during instances when the forum was used for exchanges of views in support of learning, or as a platform for social exchanges. The opportunities for, as well as challenge to, the maturity of a learning community and the socio-cognitive growth of its members are greatest when the integrity of the community is threatened by the irresponsible acts of some of its members. Not long after the establishment of the forum, some disturbing messages appeared: some students posted irresponsible messages and accusations, including attacks on the school principal; some students posted erotic websites on the forum. There erupted a lot of debate on how this should be handled. Should the forum be closed? Should students who posted those messages be banned from the forum?

With a school culture that strongly values mutual respect and trust, the school leadership decided that the incident should not lead to the closure of the discussion forum, but rather as an opportunity for educating the whole school on responsible behaviour in a cyber community. Teachers and students started posting messages on the forum to express why they found those irresponsible behaviours unacceptable. Even non-science teachers who were not involved in participating in the forum participated in the debate on how the matter should be dealt with.

It is better for us to find out why students were doing such things. Closing the forum would not help. You need to teach them what the result would be if they talk irresponsibly.

(Non-practice* ${ }^{*}$ Teacher interview

\footnotetext{
* Non-practice teachers refers to teachers in the school who were not directly involved in the innovative teaching practice.
}

(C) 2002 Blackwell Science Ltd, Journal of Computer Assisted Learning, 18, 
It is important to note that the students were able to observe, report and take pride in the growth and development of the school as a community in this process. As one of the students reported, a more mature school culture, which embraces the school community in cyber space, emerged through the discussion and debate process, moving the school a step further as a learning community in the information age: If there are new comers who talk irresponsibly and maliciously, teachers, the principal and upper form students will tell those students that they are abusing the discussion forum and it is very impolite. Eventually, a web culture is established and students know what they should and should not do in the forum. Student $L$

Later, the school gave the students the responsibility of moderating the forum. The right to delete inappropriate messages rested with the student moderators. The rationale given by the school was that the goals of establishing the forum was mainly to enhance communication for the student community, and thus it would be better for the students to take up this duty rather than the teachers.

\section{Pedagogical characteristics and learning outcomes}

While the above descriptions focused on the learning outcomes gained by the students, it is evident that those outcomes could not be dissociated from the students' learning experiences. The features most appreciated by students in these two practices were associated with the fact that the students' learning was no longer confined within the traditional classroom. Students could carry on with their project work or learning of the science topics at home, communicate with their classmates, consult their teachers, other schoolmates and alumni who may variously act as more knowledgeable others through the Internet at any time.

While all the nine innovative pedagogical practices studied in SITES M2 in Hong Kong satisfied all the case selection criteria, it was observed that the learning outcomes exhibited by the students in these nine case studies were not entirely similar. The case study described above is among the few that exhibited a full range of learning outcomes that includes the seven ICT-related knowledge management competencies mentioned in Anderson \& Plomp (2000), in addition to the sociocognitive and socio-emotional outcomes described in the previous section. A further examination of the case studies revealed that several features stood out prominently as distinguishing features found in cases that have exhibited a more diverse range of desired knowledge management competencies and which were not included in the original case selection criteria. First, these practices involved an extended learning task that spanned over a period of months rather than short learning tasks. It appears that extended tasks can provide more scope for the students to be deeply engaged personally in the learning process compared to shorter tasks, creating much better conditions for significant learning. Another common feature was that the learning tasks were personally meaningful and relevant to the students. Further enabling factors included the involvement of significant others outside of the classroom in the learning process and the availability of suitable facilitation.

Learning outcomes as resulting from induction to knowledge building

Besides the more tangible characteristics of the classroom practice, closer inspection of the SITES M2 case study data revealed finer, less tangible differences across the practices that have yet more important impacts on the learning outcomes of students. These differences relate to the teachers' awareness of and ability to focus on 
enhancing reflection and sharing of ideas among students throughout the learning process, and increasing the interdependence and interaction among students. In fact, it is apparent that the teachers and students in the above school are engaged in working with ideas and not with tasks and activities, an important distinction for knowledge building classrooms (Scardamalia, 2002). Here, knowledge building is defined according to Scardamalia \& Bereiter, (in press) as 'the production and continual improvement of ideas of value to a community, through means that increase the likelihood that what the community accomplishes will be greater than the sum of individual contributions and part of broader cultural efforts'. The authors also argue that knowledge building provides an alternative that more directly addresses the need to educate people for a world in which knowledge creation and innovation are pervasive, and that knowledge building focuses on advancing the frontiers of knowledge, which should be distinguished from shallow forms of engaging students in tasks and activities and that the learning outcome targeted is not a mere acquisition of component skills such as critical thinking, scientific method, and collaboration, which are often referred to nowadays as '21st century skills'.

Besides demonstrating a clear focus on working with ideas, the learning outcomes described above can also be identified with the socio-cognitive determinants of knowledge building as described in Scardamalia \& Bereiter (in press). These include a clear commitment to ideas being improvable objects, a respect for idea diversity, taking collective responsibility for community advancement of knowledge, and the conscious participation in and appreciation of knowledge building discourse. It is also apparent that the students have assumed epistemic agency in the learning process, deciding on goals and plans for learning as well as developing their own motivation for learning and taking up responsibility for evaluating their own communal learning. It is also heartening to note that the teachers are also appreciating this new mode of learning, demonstrating two further determinants of knowledge building in action, that of democratisation of knowledge and symmetric knowledge advancement.

In order to provide a clearer description of the distinction between working with ideas and focusing on tasks and activities, we will describe in the following section how two pedagogical practices sharing essentially the same curriculum design characteristics in the same school setting could lead to very different student learning experiences and outcomes.

\section{Teachers' facilitation focus and students' learning experience}

This is an innovative practice at the primary school level involving the use of ICT in project-based learning. The school piloted the implementation of project-based learning in the 1998-99 academic year as a form of extracurricular activities. From the following academic year (1999-2000), the school formalised project work as an integral part of the curriculum. When the SITES research team contacted the school to finalise on the specific practice to focus on for data collection in early 2001, two projects, 'My Pocket Money' and 'Understanding Myself' were underway as part of the formal curriculum in Grade 6 and Grade 4, respectively. Both projects had been designed and implemented by Teacher A as pioneer implementations of projectbased learning in the formal curriculum in the previous academic year. Thus these two projects were part of the school's efforts to scale up good practices through 
8 N. Law et al.

extending these to a wider group of teachers in order to ensure the sustainability of project-based learning. Initially, the research team found teacher B's curriculum plan for the project 'My Pocket Money' to be more interesting as it had a more comprehensive task structure that included a wider range of activities and skills, including conducting research, fund-raising and a service day in a neighborhood elderly center. However, as the weekly sessions on the projects evolved, the research team found teacher C's class to be more stimulating and leading to deeper learning outcomes. Figure 1 presents an outline of the activity flow for the two classroom practices.

Figure to be added

Fig. 1. A diagrammatic representation of the activity flow for the two classroom practices involving project based learning.

An inspection of the two activity charts in Fig. 1 reveals strong similarities as well as differences. Both project themes were very close to the everyday life of the pupils and thus have strong relevance and personal appeal to the learners. Secondly, both projects required students to collect data from other children in their school through a survey questionnaire.

In addition to having similar group tasks, the project deliverables were similar - they had to present their findings to other classes at the end of the project.

On the other hand, Fig. 1 also reveals that while both classes organised pupils into 5 groups, the task organisation, the group and student interactions designed to take place during the project process were very different. Teacher B very quickly helped the pupils to identify the key tasks and formed the groups to take care of each main task. Each group thus had a very clear task focus and goal to work on during the whole project process. While each group in this class had weekly progress meetings, the groups were essentially working independently and the assessment on individual groups focuses on how well their individual group task was accomplished. Teacher C's class, on the other hand, had many opportunities to interact. In fact, Teacher $\mathrm{C}$ created several intermediate task steps to ensure that pupils would have opportunities to interact with each other about the intermediate decisions and to increase the interdependence of the work of the various groups. These differences appeared to have arisen due to the differences in these two teachers' perception of their own role and the key purpose of facilitation. 
Facilitation focusing on task completion and skill development

In taking on the new challenge of facilitating group projects, Teacher B's pedagogical focus and attention was on the practical, task level. He wanted to ensure that students could move through the different activities and accomplish the requisite deliverables. As shown in Fig. 1, each of the groups in his class work independently of each other, going through the various stages of the project development processes such as searching for information and further refining of the problem, collect data, analyse and develop presentations were conducted entirely within each task group. Teacher B said:

My main task is to ensure that the whole project runs smoothly.

As a facilitator, Teacher B understood his own role to be that of a consultant for students, providing help and suggestions only when the pupils encountered difficulties, and that he should avoid making decisions for his students. In describing the perceived gain for the students through this classroom practice, teacher B said,

Usually their (the students') role is that of listening to the teacher's talk. But in this project, they have to ask questions and listen to the advice from others. They have to give feedback to one another at appropriate times. So the one who offers advice is not the only one who can improve. The other groups' members can make improvements too. I think the students become more mature and learn how to solve problems.

An illustration of how Teacher B facilitated the project process can be seen from the following excerpt of exchanges during a lesson when each group in the class reported on their work progress and the group (Group 1) responsible for designing the survey questions had just presented the questions they prepared:

$T$ : Is there anything else you wish to ask? Is the information asked by the questions sufficient? Is it sufficient, is the number of questions enough?

S: Sort of

$T:$ If you were to add one more question, what would you add?

$S$ : Not that I can think of at the moment.

$T$ : You can't think of any more at this moment. So you feel this is sufficient already, right? OK, Group 1, give me your list of questions. Group 2, now is your turn to present .... (T stands for teacher B and $S$ stands for a student from Group 1)

It is noteworthy that throughout this discourse, the teacher directed his questions at students in group 1 rather than the entire class. There was a noticeable absence of guidance in critical thinking and in reflecting on the learning experience when compared with Teacher C’s class.

\section{Facilitation focusing on enhancing reflection and interdependence}

While monitoring project process and helping students to work successfully to deliver the products required of them at the end is identifiably the responsibility of Teacher C, she did not see this as her main challenge or role as a facilitator. Instead, she saw her key challenge to be one of helping students to think and develop cognitively as well as metacognitively through the project process, putting the tasks and stages of project work as the contexts for helping students to develop their ability and understanding:

(The teacher) has to lead students to discuss. The teacher has to do much preparation and think of many leading questions to prompt discussion. In the discussion, it is possible that many things that are outside of the teacher's expectation occur. This is because the scope of project work is quite broad. The teacher cannot entirely predict what the students will say and what will happen. Therefore, the teacher has to do much preparation work. The teacher has to possess many skills, such as the students 
10 N. Law et al.

may ask the teachers what are genes.

The differences in these two teachers' personal emphasis and understanding in terms of their role as a facilitator can be seen clearly if we examine the following excerpt when Teacher $\mathrm{C}$ guided the discussion after group 1 had just presented their questions designed to find out whether body height and weight were related to the amount of exercise done by a person:

$T$ : Now you have all read the questions designed by Group 1. I want to see which group is the bravest to first raise questions to help them improve on their survey questions. ...You can see that Group 1's items are aimed at answering two of questions: First, is there any difference between those who do exercise and those who do not; Second, would some sports make people grow taller while others make people shorter. ...I want each group to examine the 5 questions they have designed, examine each of the five questions and think. See if find any problem with the way the questions are asked, for each of the five questions. ... . [Student $(K)$ raised her hand.] $T: \mathrm{K}$, what does your group want to say.

$K$ : The fifth question seems to be too subjective. [Some other hands were raised, wanting to speak.]

$T$ : Let's hear K explain first, you will have your turn.

$K$ : The question asked 'Do you think you have enough exercise'. This seems too

subjective ...

The class then discussed what question 5 was intended to find out and how to improve the wording of the question. Teacher $\mathrm{C}$ then spent a whole lesson guiding students to examine each other's questions and provide critical and constructive ideas to improve the items. As the questions from the various groups were to be compiled into one survey, so students were also asked to look at consistency and possible overlap across items.

Thus Teacher $\mathrm{C}$ put a much greater focus on developing the research and collaborative skills of her students. The groups had to comment and integrate their work and ideas frequently. There was a lot more interactions amongst the groups during the project development process where the whole class made decisions on all aspects of the project such that there is much greater interdependence among the students. There was also frequent use of formative peer assessment, usually conducted at the end of each stage of the project process to encourage students to think about and respond to ideas, and not just focus on the project tasks.

Consequently, though both classes have organised the project work as collaborative group-based activities, the level and intensity of interaction among the students was quite different, and the quality of the learning outcome was different. For example, in the presentation of the survey results, the grade 6 students under the guidance of Teacher B only presented the summary distribution statistics of how much pocket money students in various grade levels receive per month and the kinds of the pocket money was put to, without any discussion, interpretation or conclusion. On the other hand, the grade 4 students' (under the guidance of Teacher C) gave a much more elaborate presentation of their findings where they compared the class statistics they collected with similar statistics that the Health Department released about children of their own age group (age 9 and 10), and reported that their schoolmates tended to be taller and weighed more than other children in Hong Kong of their own age group. Further, they attempted to relate their body weight statistics to other parts of the questionnaire and, using crude bar chart comparisons, reported a positive relationship between snack-eating habits and body weight, as well as reporting that they could not find evidence for the hypothesis that body 
weight for pupils in their grade were related to the amount of water drunk, or the amount of exercise done each day.

Fostering a learning community through knowledge building discourse Reflection is an important process for learning through practice (Schon, 1983). It is interesting to note that Teacher C's focus on enhancing mutual engagement coincides with what has been preached as effective ways of fostering knowledge communities in the knowledge management literature concerned with the challenge of harnessing the tacit dimension of knowledge e.g. Wenger, 2000. The tacit knowledge refers to what Sallis (2002) describes as 'knowledge that is felt': hunches, insights, intuitions, feelings, imagery and emotions, personal knowledge that are 'developed through social interactions, power plays, teamwork, friendship and corporate politics'. Teacher C's focus also coincides with the findings on the importance of cultivating constructive criticism and engendering interdependence as the keys to effective facilitation of collaborative project based learning reported in Law et al. (2000).

\section{Preparation for lifelong learning requires paradigmatic changes in teaching}

If the goal of education is to prepare students for life in a fast changing world where the shelf life of knowledge is getting ever shorter and the ability to work collaboratively with others on new problems is essential for effective functioning in the workplace, the abilities to be cultivated through schooling becomes very different from those found in the traditional curriculum. Rather than ensuring that students master a set of core contents and skills, schools need to prepare students who are able to communicate, think and continue to learn throughout their lives and who can work productively and effectively with others through negotiations and compromises (Gardner, 1991; Sarason, 1990). Students need to have the experience of engaging in collaborative knowledge building, in collectively advancing the frontiers of knowledge as they experience it. To bring about such learning requires new roles for teachers and new teaching strategies and practices. Schooling should become a process of enculturation, guiding students to build on their 'natural human tendency to play creatively with ideas, and expands it to the unnatural human capacity to exceed the boundaries of what is known and knowable - to exceed expectations rather than settle into routines' (Scardamalia \& Bereiter, in press). This is far different from the general realities of current classrooms which are designed for achieving the educational goals of the modern industrial age:

The traditional classroom ...... is singularly ill suited to producing lifelong learners: 'Right now, you've got 30 little workers who come into a room, sit in rows, follow instructions from a boss, and can't talk to one another. School is the last time they'll ever see that model'. (Corcoran, 1993, cited in Ogilvy et al., 1998)

The use of ICT to support teaching and learning across the curriculum may or may not support the development of lifelong learning abilities. In fact, it is anticipated that current usage of ICT in educational settings is more likely to be in the form of deploying new technology for the delivery of old pedagogical practices (Plomp et al., 1996). The development of new pedagogies need nurturing and encouragement. It is in recognition of the importance of identifying and studying such new pedagogies that uses ICT that the SITES M2 study was developed. 


\section{Knowledge building discourse and 21st century learning outcomes}

Findings from the present study indicate that, in order to encourage the development of lifelong learning abilities of students for participation in a learning community, the design of ICT-using classroom practices must not just focus on task characteristics and the cognitive learning outcomes to be targeted. Of greater importance is the pedagogical focus of the teacher, whether s/he has a focus on encouraging students to work with ideas, on supporting the reflective approach to the learning tasks and on developing an empowering learning culture. In short, the learning process should fosters the growth and development of the socio-cognitive determinants of knowledge building (Scardamalia \& Bereiter, in press ). For the evaluation of learning outcomes, there are strong arguments for assessing the affective and attitudinal dimensions of learning as a socio-cognitive activity. These are probably more critical to the longer-term ability of the learner to cope with the demands of lifelong learning in a knowledge society.

\section{References}

Anderson, R. \& Plomp, T. (2000) ICT Knowledge Management Competencies - a plan for SITES-M3. (Research proposal). IEA, Amsterdam.

Bereiter, C. \& Scardamalia, M. (1998) Beyond Bloom's Taxonomy: Rethinking knowledge for the knowledge age. In Fullan, M. (Ed.) The International Handbook of Educational Change . pp. 675-692. Kluwer Academic Publishers: Dordrecht, The Netherlands.

Corcoran, E. (1993) Why Kids Love Computer Nets. Fortune, 104. (September 20)

Gardner, H. (1991) The Unschooled Mind. Basic Books, New York.

Ireland, I.S. (1999) Building a Capacity for Change: Lifelong Learning in the Information Society. Stationery Office, Government of Ireland, Dublin.

KME (2000) 2000 Adapting Education to the Information Age: A White Paper. Korean Ministry of Education, Education \& Research Information Service, Seoul.

Kozma, R. \& Schank, P. (1998) Connecting with the 21st Century: Technology in support of educational reform. In Learning with Technology (ed. C. Dede) pp. 3-27, Association for Supervision and Curriculum Development: Alexandria, VA.

Law, N., Yuen, H.K. \& Ma, M. (2000) What Happens in Project-based Learning. Paper presented at the CITE Colloquium 2000. University of Hong Kong, Hong Kong.

OECD (2000) Schooling for Tomorrow: Learning to Bridge the Digital Divide. OECD, Paris.

Ogilvy, J. (1998) Education \& Community: Four Scenarios for the Future of Public Education. Global Business Network, (22-2-98).

PCAST (President's Committee of Advisors on Science and Technology Panel on Educational Technology) (1997) Report to the President on the Use of Technology to Strengthen K-12 Education in the United States. http://www.ostp.gov/PCAST/k12ed.html, accessed 10/7/2002

Pelgrum, H. \& Anderson, R. (eds.) (1999) ICT and the Emerging Paradigm for Life Long Learning. IEA, Amsterdam.

Plomp, T., ten Brummelhuis, A. \& Rapmund, R. (eds.) (1996) Teaching and Learning for the Future. Committee on Multimedia in Teacher Training, Dutch Ministry of Education, Den Haag.

Sallis, E.J. \& G. (2002) Knowledge Management in Education: Enhancing Learning and Education. Kogan Page, London.

Sarason, S. (1990) The Predictable Failure of Educational Reform. Jossey-Bass, San Francisco, CA. 
Scardamalia, M. (2002) Collective Cognitive Responsibility for the Advancement of Knowledge. IKIT, OISE., University of Toronto. Available http://db.ikit.org:37495 (accessed 2002, July 11, 2002).

Scardamalia, M. \& Bereiter, C. (in press) Knowledge Building. In Encyclopedia of Education, (editors/pages???). Macmillan Reference, New York.

Schon, D. (1983) The Reflective Practitioner: How Professionals Think in Action. Basic Books, New York.

Seltzer, K. \& Bentley, T. (2001) The Creative Age: Knowledge and Skills for the New Economy. Demos, London.

SME (1997) IT Masterplan for Education. Singapore Ministry of Education, Singapore. Wenger, E. (2000) Communities of Practice. Cambridge University Press, Cambridge. 


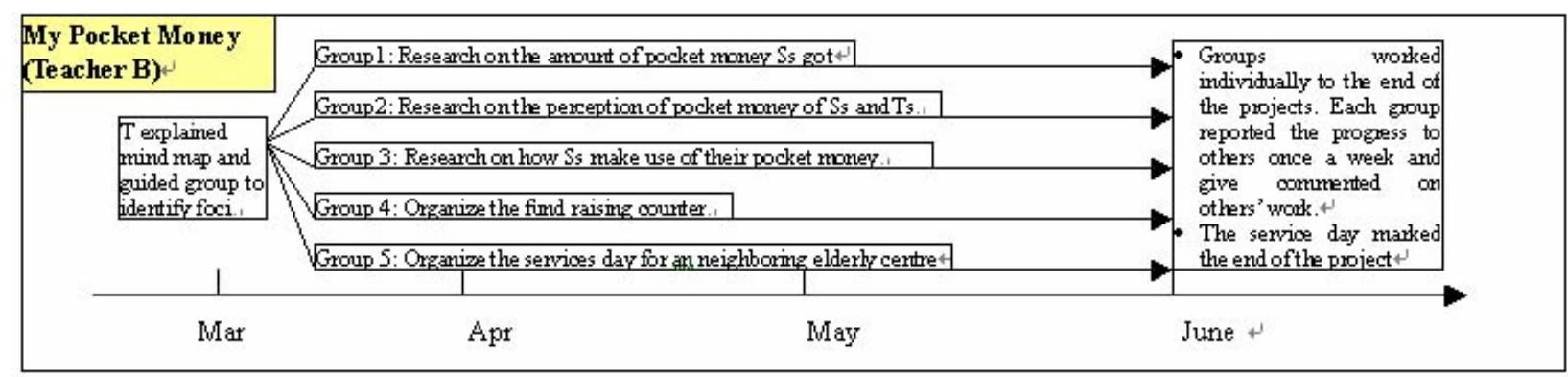

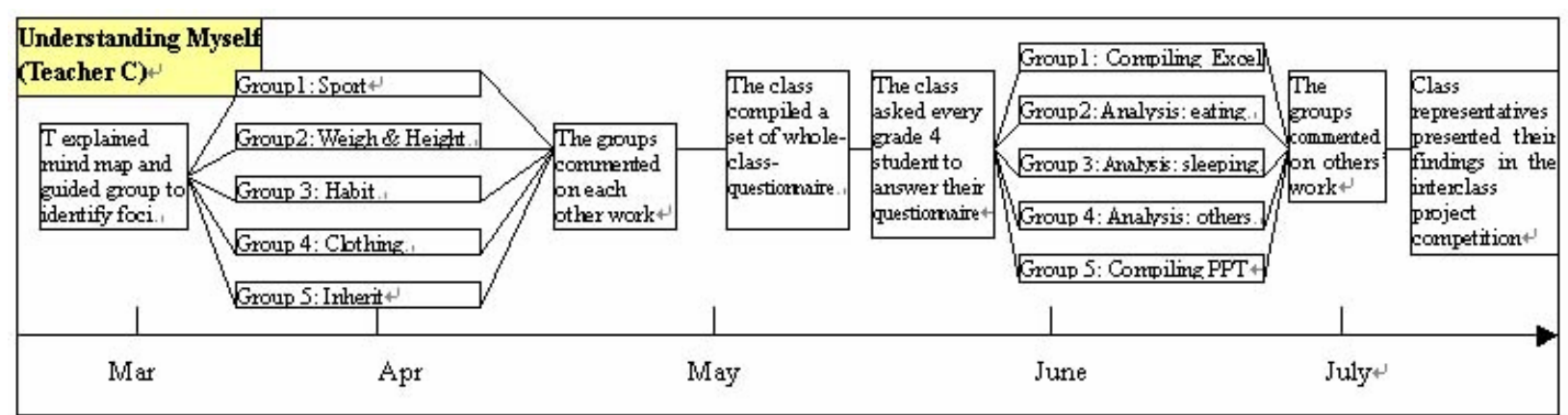

Figure 1. A diagrammatic representation of the activity flow for the two classroom practices involving project based learning. 